\title{
PRE-JORDAN ALGEBRAS
}

\author{
DONGPING HOU, XIANG NI and CHENGMING BAI*
}

\begin{abstract}
The purpose of this paper is to introduce and study a notion of pre-Jordan algebra. Pre-Jordan algebras are regarded as the underlying algebraic structures of the Jordan algebras with a nondegenerate symplectic form. They are the algebraic structures behind the Jordan Yang-Baxter equation and Rota-Baxter operators in terms of $\mathscr{O}$-operators of Jordan algebras introduced in this paper. Pre-Jordan algebras are analogues for Jordan algebras of pre-Lie algebras and fit into a bigger framework with a close relationship with dendriform algebras. The anticommutator of a preJordan algebra is a Jordan algebra and the left multiplication operators give a representation of the Jordan algebra, which is the beauty of such a structure. Furthermore, we introduce a notion of $\mathscr{O}$-operator of a pre-Jordan algebra which gives an analogue of the classical Yang-Baxter equation in a pre-Jordan algebra.
\end{abstract}

\section{Introduction}

\subsection{Motivations}

Jordan algebras were first studied in the 1930s in the context of axiomatic quantum mechanics ([6]) and appeared in many areas of mathematics like differential geometry ([30], [19], [35], [37], [44]), Lie theory ([31], [34]) and analysis ([37], [48]). A Jordan algebra can be regarded as an "opposite" of a Lie algebra in the sense that the commutator of an associative algebra is a Lie algebra and the anticommutator of an associative algebra is a Jordan algebra, although not every Jordan algebra is isomorphic to the anticommutator of an associative algebra (such a Jordan algebra is called special, otherwise, it is called exceptional).

In this paper, we introduce a notion of pre-Jordan algebra, which is closely related to Jordan algebras, from the following motivations in different (closely related) fields.

(1) Jordan Yang-Baxter equation and $\mathscr{O}$-operators. The notion of Jordan D-bialgebra was introduced by Zhelyabin in [50] as an analogue of a Lie bialgebra (also see [51], [52],[53]). A class of Jordan D-bialgebras (coboundary cases) are obtained from the solutions of an algebraic equation in a Jordan algebra, which is an analogue of the classical Yang-Baxter equation (CYBE)

\footnotetext{
* Corresponding author.

Received 7 January 2010, in final form 20 April 2010.
} 
in a Lie algebra ([51], [53]). It is called Jordan Yang-Baxter equation (JYBE) for convenience. The CYBE plays an important role in integrable systems and quantum groups ([17] and the references therein). The original forms of both the CYBE and the JYBE are given in the tensor forms and it is natural to consider their operator forms which are the linear transformations corresponding to the elements in the tensor product spaces satisfying the equations.

In the case of Lie algebras, it was Semenov-Tian-Shansky who first introduced certain operator form of the CYBE ([47]). Later Kupershmidt introduced a notion of $\mathscr{O}$-operator of a Lie algebra as a generalization of (the operator form of) the CYBE in a Lie algebra ([36]). Nevertheless, the $\mathscr{O}$-operators of Lie algebras play more interesting roles in the study of the CYBE. In fact, a skew-symmetric solution of the CYBE is exactly a special $\mathscr{O}$-operator (associated to the coadjoint representation) and more importantly, there are a kind of algebraic structures behind the $\mathscr{O}$-operators of Lie algebras and the related CYBE, namely, pre-Lie algebras, in the following sense: the $\mathcal{O}$-operators of Lie algebras provide a direct relationship between Lie algebras and pre-Lie algebras and in the invertible cases, they provide a necessary and sufficient condition for the existence of a compatible pre-Lie algebra structure on a Lie algebra; as an immediate consequence, there are some solutions of the CYBE in certain Lie algebras obtained from pre-Lie algebras ([8]).

In order to understand the operator forms of the JYBE and the JYBE itself well, we introduce a notion of $\mathscr{O}$-operator of a Jordan algebra in this paper and we will show that it plays a similar role of the $\mathscr{O}$-operator of a Lie algebra. Then it is natural to ask what algebraic structures behind the $\mathscr{O}$-operators of Jordan algebras and the related JYBE? The answer is pre-Jordan algebras!

(2) Rota-Baxter operators. Another kind of $\mathscr{O}$-operators of Jordan algebras (associated to the regular modules) are Rota-Baxter operators. Rota-Baxter operators (on associative algebras) were introduced by G. Baxter ([12]) in 1960. The importance of these operators were realized by G.-C. Rota in combinatorics ([45]). Since then, many applications of Rota-Baxter operators have been discovered in various areas of mathematics and mathematical physics (see for instance [7], [14], [20], [22]). Obviously they are well-defined on any algebra. Recently, Rota-Baxter operators were found to provide an approach of constructing certain type of algebras with richer structures from a known type of algebras ([25]), such as a Rota-Baxter operator on an associative algebra is used to construct a dendriform algebra ([2]), a Rota-Baxter operator on a dendriform algebra or a pair of commutating Rota-Baxter operators on an associative algebra is used to construct a quadri-algebra ([4]). As an independent topic, with the above approach and idea, the algebraic structure from a Rota-Baxter operator of a Jordan algebra is exactly a pre-Jordan algebra, although it is 
an immediate consequence from the relationship between pre-Jordan algebras and Jordan algebras in terms of $\mathscr{O}$-operators of Jordan algebras.

(3) Symplectic structures on Jordan algebras. There is a correspondence between the invertible skew-symmetric solutions of the JYBE and the nondegenerate (skew-symmetric) symplectic form on a Jordan algebra ([52]). It is an analogue of Drinfeld's observation on the relationship between the invertible (skew-symmetric) solutions of the CYBE and the symplectic forms on a Lie algebra ([21]). We will show that there exists a natural pre-Jordan algebra structure on a Jordan algebra with a nondegenerate (skew-symmetric) symplectic form. Conversely, in some subsequent works, we will see that pre-Jordan algebras play essential roles in certain constructions of Jordan algebras with a nondegenerate (skew-symmetric) symplectic form ([29]). In this sense, like pre-Lie algebras regarded as the underlying algebraic structures of the symplectic Lie algebras ([18]), pre-Jordan algebras are regarded as the underlying algebraic structures of the Jordan algebras with a nondegenerate (skew-symmetric) symplectic form.

(4) Pre-Lie algebras. As we have mentioned in (1), pre-Jordan algebras are certain analogues of pre-Lie algebras in terms of $\mathcal{O}$-operators. Nevertheless, there are more analogues between them, even in the algebraic structures themselves. In fact, except for the mentioned appearance in the study of the CYBE and symplectic Lie groups and Lie algebras, pre-Lie algebras (or under other names like left-symmetric algebras and quasi-associative algebras) are a class of natural algebraic systems appearing in many fields in mathematics and mathematical physics such as convex homogenous cones, affine manifolds and affine structures on Lie groups, deformation of associative algebras, certain integrable systems, combinatorics, quantum field theory and vertex algebras (see the survey article [13] and the references therein).

The beauty of a pre-Lie algebra is that its commutator is a Lie algebra and its left multiplication operators give a representation of the Lie algebra. Since the Jordan algebras are the "opposite" of the Lie algebras, it is natural and interesting enough from a pure algebraic point of view to consider the similar algebraic structure for a Jordan algebra, that is, the algebraic structure satisfies that its anticommutator is a Jordan algebra and its left multiplication operators give a representation of the Jordan algebra, which is exactly the definition of a pre-Jordan algebra. In fact, the notion of "pre-Jordan algebra" is given because of this reason. As pointed out in [16], the pre-Lie algebras "deserve more attention than they have been given". We believe that pre-Jordan algebras might do so too.

(5) Dendriform algebras. In fact, pre-Jordan algebras fit into a bigger framework. Recall that a dendriform algebra is equipped with an associative 
product which can be written as a linear combination of nonassociative compositions. It was introduced by Loday ([39]) in 1995 with motivation from algebraic $K$-theory and has been studied quite extensively with connections to several areas in mathematics and physics, including operads ([41]), homology ([27], [28]), Hopf algebras ([15], [43]), Lie and Leibniz algebras ([28]), combinatorics ([42]), arithmetic([40]) and quantum field theory ([26]). Also see [25] and the references therein.

Moreover, due to Chapoton ([15], also see [1], [2], [3]), there is a close relationship among Lie algebras, associative algebras, pre-Lie algebras and dendriform algebras as follows (in the sense of commutative diagram of categories):

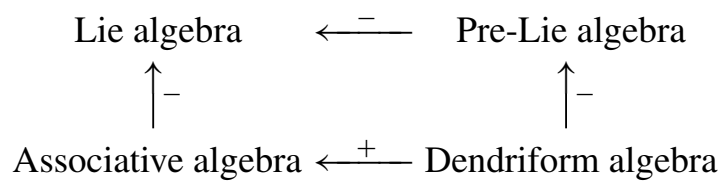

Explicitly, let $(A, \succ, \prec)$ be a dendriform algebra, then the operation given by

$$
x * y=x \succ y+x \prec y, \quad \forall x, y \in A
$$

defines an associative algebra and the operation

$$
x \bullet y=x \succ y-y \prec x, \quad \forall x, y \in A
$$

defines a pre-Lie algebra. The other two "-_" operations in the diagram (1.1) are the commutators.

There are two related questions arising naturally. One is about the dendriform algebras themselves: whether there are some algebraic structures corres-

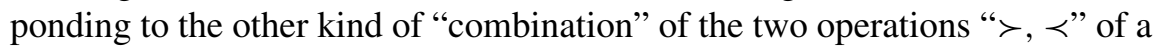
dendriform algebra, for example, what algebraic structure $(A, \cdot)$ should satisfy

$$
x \cdot y=x \succ y+y \succ x, \quad \forall x, y \in A \text {. }
$$

On the other hand, due to the relationship between associative algebras, Lie algebras and Jordan algebras, it is also natural to consider the "opposite" of the commutative diagram (1.1), that is, find a suitable algebra structure in the place of "pre-Lie algebra" when the "Lie algebra" is replaced by the "Jordan algebra" and the commutator is replaced by the anticommutator.

We will show that the pre-Jordan algebra is a good candidate to answer the 
above two questions, that is, there is the following commutative diagram:

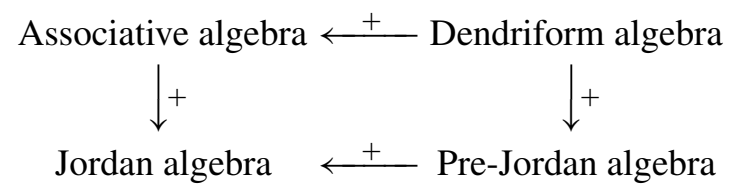

In this sense, a pre-Jordan algebra is not only an analogue of a pre-Lie algebra again which is consistent with the previous motivations, but can also be regarded as an "opposite" of a pre-Lie algebra. Moreover, the above commutative diagram is also consistent with the construction from the $\mathscr{O}$-operators including the Rota-Baxter operators.

\subsection{Layout of the paper}

The paper is organized as follows. In Section 2, we give some fundamental results on Jordan algebras and the JYBE. In Section 3, we introduce the notion of $\mathscr{O}$-operator of a Jordan algebra and then give a further study on the JYBE. In Section 4, we introduce the notion of pre-Jordan algebra and study the relationships with Jordan algebras, the JYBE, the Jordan algebras with a nondegenerate (skew-symmetric) symplectic form and dendriform algebras in terms of $\mathscr{O}$-operators of Jordan algebras. In Section 5, we introduce a notion of $\mathcal{O}$-operator of a pre-Jordan algebra. An analogue of the CYBE and the JYBE in a pre-Jordan algebra and some bilinear forms on pre-Jordan algebras satisfying certain conditions are given. In Section 6, we introduce what we call Jordan analogues of Loday algebras as a generalization of the study of pre-Jordan algebras.

\subsection{Notations}

Throughout this paper, all algebras are finite-dimensional and over a field $\mathrm{F}$ of characteristic zero. Let $(A, \cdot)$ be an algebra. We use the following notations.

(1) Let $L .(x)$ and $R .(x)$ denote the left and right multiplication operator respectively, that is, $L .(x)(y)=R .(y)(x))=x \cdot y$ for any $x, y \in A$ (or simply by $L(x)$ and $R(x)$ respectively without confusion). Moreover, let $L ., R .: A \rightarrow$ $\operatorname{gl}(A)$ be two linear maps with $x \mapsto L .(x)$ and $x \mapsto R$. $(x)$ respectively.

(2) Let $r=\sum_{i} a_{i} \otimes b_{i} \in A \otimes A$. Set

$$
r_{12}=\sum_{i} a_{i} \otimes b_{i} \otimes 1, \quad r_{13}=\sum_{i} a_{i} \otimes 1 \otimes b_{i}, \quad r_{23}=\sum_{i} 1 \otimes a_{i} \otimes b_{i},
$$

where 1 is a unit element if $(A, \cdot)$ is unital or a symbol playing a similar role of the unit for the nonunital cases. The operation between two $r \mathrm{~s}$ is in an obvious 
way. For example,

$$
\begin{aligned}
& r_{12} \cdot r_{13}=\sum_{i, j} a_{i} \cdot a_{j} \otimes b_{i} \otimes b_{j}, \\
& r_{13} \cdot r_{23}=\sum_{i, j} a_{i} \otimes a_{j} \otimes b_{i} \cdot b_{j}, \\
& r_{23} \cdot r_{12}=\sum_{i, j} a_{j} \otimes a_{i} \cdot b_{j} \otimes b_{i} .
\end{aligned}
$$

Note that Eq. (1.7) is independent of the existence of the unit.

(3) Let $V$ be a vector space. Let $\sigma: V \otimes V \rightarrow V \otimes V$ be the exchanging operator defined as

$$
\sigma(x \otimes y)=y \otimes x, \quad \forall x, y \in V .
$$

A tensor $r \in V \otimes V$ is called symmetric (skew-symmetric respectively) if $r=\sigma(r)(r=-\sigma(r)$ respectively). On the other hand, any $r \in V \otimes V$ can be identified as a linear map from the dual space $V^{*}$ to $V$ in the following way:

$$
\left\langle u^{*}, r\left(v^{*}\right)\right\rangle=\left\langle u^{*} \otimes v^{*}, r\right\rangle, \quad \forall u^{*}, v^{*} \in V^{*},
$$

where $\langle$,$\rangle is the ordinary pairing between the vector space V$ and the dual space $V^{*}$. The tensor $r \in V \otimes V$ is called nondegenerate if the above induced linear map is invertible. Moreover, any invertible linear map $T: V^{*} \rightarrow V$ induces a nondegenerate bilinear form $\mathscr{B}($, ) on $V$ by

$$
\mathscr{B}(u, v)=\left\langle T^{-1}(u), v\right\rangle, \quad \forall u, v \in V .
$$

Furthermore, $T$ is called symmetric (skew-symmetric respectively) if the induced bilinear form $\mathscr{B}$ is symmetric (skew-symmetric respectively). Since $T$ can be also regarded as an element in $V \otimes V$ by Eq. (1.9), both of the symmetries or skew-symmetries of $T$ coincide obviously.

(4) Let $V$ be a vector space. There are two natural symmetric and skewsymmetric bilinear forms $\mathscr{B}$ and $\omega$ on the vector space $V \oplus V^{*}$ given by (for any $\left.x, y \in V, a^{*}, b^{*} \in V^{*}\right)$

$$
\begin{aligned}
\mathscr{B}\left(x+a^{*}, y+b^{*}\right) & =\left\langle a^{*}, y\right\rangle+\left\langle x, b^{*}\right\rangle, \\
\omega\left(x+a^{*}, y+b^{*}\right) & =\left\langle a^{*}, y\right\rangle-\left\langle x, b^{*}\right\rangle,
\end{aligned}
$$

respectively.

(5) Let $V_{1}, V_{2}$ be two vector spaces and $T: V_{1} \rightarrow V_{2}$ be a linear map. Denote the dual (linear) map by $T^{*}: V_{2}^{*} \rightarrow V_{1}^{*}$ given by

$$
\left\langle T^{*}\left(v_{2}^{*}\right), v_{1}\right\rangle=\left\langle v_{2}^{*}, T\left(v_{1}\right)\right\rangle, \quad \forall v_{1} \in V_{1}, v_{2}^{*} \in V_{2}^{*} .
$$


On the other hand, $T$ can be identified as an element in $V_{2} \otimes V_{1}^{*}$ by

$$
\left\langle v_{2}^{*} \otimes v_{1}, T\right\rangle=\left\langle v_{2}^{*}, T\left(v_{1}\right)\right\rangle, \quad \forall v_{1} \in V_{1}, v_{2}^{*} \in V_{2}^{*} \text {. }
$$

Note that Eq. (1.9) is exactly the case $V_{1}=V_{2}^{*}$ of Eq. (1.14). Moreover, in the above sense, any linear map $T: V_{1} \rightarrow V_{2}$ is obviously an element in $\left(V_{2} \oplus V_{1}^{*}\right) \otimes\left(V_{2} \oplus V_{1}^{*}\right)$.

(6) Let $V$ be a vector space. For any linear map $\rho: A \rightarrow \operatorname{gl}(V)$, define a linear map $\rho^{*}: A \rightarrow \operatorname{gl}\left(V^{*}\right)$ by

$$
\left\langle\rho^{*}(x) v^{*}, u\right\rangle=\left\langle v^{*}, \rho(x) u\right\rangle, \quad \forall x \in A, u \in V, v^{*} \in V^{*} .
$$

Note that in this case, $\rho^{*}$ is different from the one given by Eq. (1.13) which regards $\mathrm{gl}(V)$ as a vector space, too.

\section{Jordan algebras and Jordan Yang-Baxter equation}

\subsection{Some basic results on Jordan algebras}

DEFINITION 2.1. A Jordan algebra is a vector space $J$ equipped with a commutative binary operation $(x, y) \rightarrow x \circ y$ satisfying the following Jordan identity:

$$
((x \circ x) \circ y) \circ x=(x \circ x) \circ(y \circ x), \quad \forall x, y \in J .
$$

REMARK 2.2. (1) If $(A, *)$ is an associative algebra, then the operation given by

$$
x \circ y=x * y+y * x, \quad \forall x, y \in A,
$$

defines a Jordan algebra structure on $A$. Such a Jordan algebra is a special Jordan algebra.

(2) When ch $\mathrm{F} \neq 2$ and 3, it was pointed out in [5] that the Jordan identity (2.1) is equivalent to the following identity (for any $x, y, z, u \in J$ )

$$
\begin{aligned}
& ((x \circ y) \circ u) \circ z+((y \circ z) \circ u) \circ x+((z \circ x) \circ u) \circ y \\
& =(x \circ y) \circ(u \circ z)+(y \circ z) \circ(u \circ x)+(z \circ x) \circ(u \circ y),
\end{aligned}
$$

or equivalently,

$$
(x \circ y, u, z)+(y \circ z, u, x)+(z \circ x, u, y)=0, \quad \forall x, y, z, u \in J,
$$

where $(x, y, z)=(x \circ y) \circ z-x \circ(y \circ z)$ is the associator. 
Definition 2.3. Let $(J, \circ)$ be a Jordan algebra and $V$ be a vector space. A linear map $\rho: J \rightarrow \operatorname{gl}(V)$ is called a representation (or a module) of $(J, \circ)$ if (for any $x, y, z \in J$ )

$$
\begin{aligned}
& {[\rho(x), \rho(y \circ z)]+[\rho(y), \rho(z \circ x)]+[\rho(z), \rho(x \circ y)]=0} \\
& \rho(x) \rho(y) \rho(z)+\rho(z) \rho(y) \rho(x)+\rho((x \circ z) \circ y) \\
& =\rho(x) \rho(y \circ z)+\rho(y) \rho(z \circ x)+\rho(z) \rho(x \circ y),
\end{aligned}
$$

where $[$,$] is the commutator. We denote it by (\rho, V)$ or simply $\rho$.

In fact, $(\rho, V)$ is a module of a Jordan algebra $(J, \circ)$ if and only if there exists a Jordan algebra structure on the direct sum $J \oplus V$ (the semi-direct sum) of the underlying vector spaces of $J$ and $V$ given by

$$
(x+u) \circ(y+v)=x \circ y+\rho(x) v+\rho(y) u, \quad \forall x, y \in J, u, v \in V .
$$

We denote it by $J \ltimes_{\rho} V$ or simply $J \ltimes V$.

Remark 2.4. Let $(J, \circ)$ be a Jordan algebra. If a linear map $\rho: J \rightarrow \operatorname{gl}(V)$ satisfies

$$
\rho(x \circ y)=\rho(x) \rho(y)+\rho(y) \rho(x), \quad \forall x, y \in J,
$$

then $\rho$ also satisfies Eqs. (2.5) and (2.6). So it is a representation of $(J, \circ)$ which is called a special representation.

Proposition-Definition 2.5 ([32]). Let $(\rho, V)$ be a representation of a Jordan algebra $(J, \circ)$. Then $\left(\rho^{*}, V^{*}\right)$ is a representation of $(J, \circ)$, which is called the dual representation of $(\rho, V)$.

Proposition-Definition 2.6 ([33]). Let $(J, o)$ be a Jordan algebra. For any $x \in J$, let $\operatorname{rg}(x)$ denote the left (right) multiplication operator, that is, $\operatorname{rg}(x)(y)=x \circ y=y \circ x$ for any $y \in J$. Let $\mathrm{rg}: J \rightarrow \operatorname{gl}(J)$ be a linear map with $x \mapsto \operatorname{rg}(x)$. Then both $(\mathrm{rg}, J)$ and $\left(\mathrm{rg}^{*}, J^{*}\right)$ are representations of $(J, \circ)$. The former is called the regular representation of $J$.

Definition 2.7. Let $(J, \circ)$ be a Jordan algebra. A symmetric bilinear form $\mathscr{B}$ on $J$ is called invariant if

$$
\mathscr{B}(x \circ y, z)=B(x, y \circ z), \quad \forall x, y, z \in J .
$$




\subsection{Jordan D-bialgebras and Jordan Yang-Baxter equation}

Definition 2.8 ([50]). Let $(J, \circ)$ be a Jordan algebra. A Jordan D-bialgebra structure on $J$ is a linear map $\Delta: J \rightarrow J \otimes J$ which is called co-multiplication such that $\Delta^{*}: J^{*} \otimes J^{*} \rightarrow J^{*}$ defines a Jordan algebra structure on $J^{*}$ and the following three conditions hold (for any $x, y \in J$ )

$$
(\Delta \otimes \mathrm{id}-\mathrm{id} \otimes \Delta) \Delta\left(x^{2}\right)
$$

$$
\begin{aligned}
= & 2(1 \otimes x \otimes 1) \circ(\Delta \otimes \mathrm{id}-\mathrm{id} \otimes \Delta) \Delta(x) \\
& +2(1 \otimes 1 \otimes x-x \otimes 1 \otimes 1) \circ(\mathrm{id} \otimes \sigma)(\Delta \otimes \mathrm{id}) \Delta(x) \\
& +2(\Delta(x) \otimes 1-1 \otimes \Delta(x)) \circ((\mathrm{id} \otimes \sigma)(\Delta(x) \otimes 1)),
\end{aligned}
$$

$(\Delta \otimes \mathrm{id}+\mathrm{id} \otimes \Delta+(\sigma \otimes \mathrm{id})(\mathrm{id} \otimes \Delta))((1 \otimes x+x \otimes 1) \circ \Delta(x))$

$$
\begin{aligned}
= & 2(1 \otimes x \otimes 1) \circ((\mathrm{id} \otimes \Delta) \Delta(x)) \\
& +2(x \otimes 1 \otimes 1) \circ((\mathrm{id} \otimes \sigma)(\Delta \otimes \mathrm{id}) \Delta(x)) \\
& +2(1 \otimes \Delta(x)) \circ((\mathrm{id} \otimes \sigma)(\Delta(x) \otimes 1))+(\Delta \otimes \mathrm{id}) \Delta\left(x^{2}\right),
\end{aligned}
$$

$$
\begin{aligned}
& \Delta\left(x^{2} \circ y\right)-\Delta\left(x^{2}\right) \circ(y \otimes 1)-\Delta(y) \circ\left(1 \otimes x^{2}\right)+2 \Delta(y)(x \otimes x) \\
&= 2 \Delta(x \circ y) \circ(x \otimes 1)-2(\Delta(x) \circ(y \otimes 1)) \circ(x \otimes 1) \\
&-2(\Delta(x) \circ(1 \otimes y)) \circ(1 \otimes x)+2 \Delta(x) \circ(1 \otimes(x \circ y)),
\end{aligned}
$$

where 1 is the unit in the universal enveloping algebra $U(J)$. We denote it by $(J, \Delta)$.

REMARK 2.9. According to [50] and [53], a Jordan D-bialgebra $(J, \Delta)$ is equivalent to the following structure: there is a Jordan algebra structure on the direct sum $J \oplus J^{*}$ of the underlying vector spaces of $J$ and $J^{*}$ such that both $J$ and $J^{*}$ are subalgebras and the symmetric bilinear form on $J \oplus J^{*}$ given by Eq. (1.11) is invariant.

Proposition 2.10 ([53]). Let $(J, \circ)$ be a Jordan algebra and $r \in J \otimes J$. Set

$$
\Delta_{r}(x)=(\operatorname{rg}(x) \otimes \mathrm{id}-\mathrm{id} \otimes \operatorname{rg}(x)) r, \quad \forall x \in J .
$$

If $r$ is skew-symmetric and $r$ satisfies

$$
r_{12} \circ r_{13}+r_{13} \circ r_{23}-r_{12} \circ r_{23}=0
$$

then $\left(J, \Delta_{r}\right)$ is a Jordan D-bialgebra. 
Definition 2.11. Let $(J, \circ)$ be a Jordan algebra and $r \in J \otimes J$. Eq. (2.14) was called "Jordan analogue" of the classical Yang-Baxter equation in [51] and [53]. We call it Jordan Yang-Baxter equation (JYBE) in $J$ for convenience.

Proposition 2.12 ([52]). Let $(J$, o) be a Jordan algebra and $r \in J \otimes J$. Suppose that $r$ is skew-symmetric and nondegenerate. Then $r$ is a solution of JYBE in $J$ if and only if the bilinear form $\omega$ induced by $r$ through Eq. (1.10) satisfies

$$
\omega(x \circ y, z)+\omega(y \circ z, x)+\omega(z \circ x, y)=0, \quad \forall x, y, z \in J .
$$

Definition 2.13 ([52]). A symplectic form $\omega$ on a Jordan algebra $(J, \circ)$ is a skew-symmetric bilinear form satisfying Eq. (2.15).

Remark 2.14. In some references (for example, [11]), a symplectic form on a Jordan algebra is assumed to be nondegenerate.

\section{3. $\mathscr{O}$-operators of Jordan algebras and Jordan Yang-Baxter equation}

Proposition 3.1. Let $(J, \circ)$ be a Jordan algebra and $r \in J \otimes J$ be skewsymmetric. Then $r$ is a solution of JYBE in $J$ if and only if $r$ satisfies

$$
r\left(a^{*}\right) \circ r\left(b^{*}\right)=r\left(\operatorname{rg}^{*}\left(r\left(a^{*}\right)\right) b^{*}+\operatorname{rg}^{*}\left(r\left(b^{*}\right)\right) a^{*}\right), \quad \forall a^{*}, b^{*} \in J^{*} .
$$

Proof. Let $\left\{e_{1}, \ldots, e_{n}\right\}$ be a basis of $J$ and $\left\{e_{1}^{*}, \ldots, e_{n}^{*}\right\}$ be the dual basis. Suppose that $e_{i} \circ e_{j}=\sum_{k=1}^{n} c_{i j}^{k} e_{k}$ and $r=\sum_{i, j=1}^{n} a_{i j} e_{i} \otimes e_{j}, a_{i j}=-a_{j i}$. Hence $r\left(e_{i}^{*}\right)=\sum_{k=1}^{n} a_{k i} e_{k}=-\sum_{k=1}^{n} a_{i k} e_{k}$. Then $r$ is a solution of JYBE in $J$ if and only if (for any $i, j, k$ )

$$
\sum_{t, l=1}^{n}\left\{c_{t l}^{i} a_{t j} a_{l k}-c_{t l}^{j} a_{i t} a_{l k}+c_{t l}^{k} a_{i t} a_{j l}\right\}=0 .
$$

The left-hand side of the above equation is just the coefficient of $e_{k}$ in the following equation

$$
-r\left(\operatorname{rg}^{*}\left(r\left(e_{j}^{*}\right)\right) e_{i}^{*}+\operatorname{rg}^{*}\left(r\left(e_{i}^{*}\right)\right) e_{j}^{*}\right)+r\left(e_{i}^{*}\right) \circ r\left(e_{j}^{*}\right) .
$$

Therefore the conclusion holds.

Definition 3.2. Let $(J, \circ)$ be a Jordan algebra. A Rota-Baxter operator (of weight zero) on $J$ is a linear map $R: J \rightarrow J$ satisfying

$$
R(x) \circ R(y)=R(R(x) \circ y+x \circ R(y)), \quad \forall x, y \in J .
$$


REMARK 3.3. In the case of associative algebras, a Rota-Baxter operator of weight $\lambda \in \mathrm{F}$ on an associative algebra $(A, \cdot)$ is a linear map $R: A \rightarrow A$ satisfying

$$
R(x) \cdot R(y)=R(R(x) \cdot y)+R(x \cdot R(y))+\lambda R(x \cdot y), \quad \forall x, y \in A .
$$

This notion has an analogue in the context of Jordan algebras too, but, in this paper, we only use Rota-Baxter operators of Jordan algebras of weight zero.

COROLlary 3.4. Let $(J, \circ)$ be a Jordan algebra and $r \in J \otimes J$ be skewsymmetric. Suppose that there is a symmetric nondegenerate invariant bilinear form $\mathscr{B}$ on $J$. Let $\varphi: J \rightarrow J^{*}$ be a linear map given by $\langle\varphi(x), y\rangle=\mathscr{B}(x, y)$ for any $x, y \in J$. Then $r$ is a solution of JYBE in $J$ if and only if $r \varphi$ is a Rota-Baxter operator (of weight zero) on $\mathrm{J}$.

Proof. In fact, $\varphi(\operatorname{rg}(x) y)=\operatorname{rg}^{*}(x) \varphi(y)$ for any $x, y \in J$ since

$$
\begin{aligned}
\langle\varphi(\operatorname{rg}(x) y), z\rangle & =\mathscr{B}(x \circ y, z)=\mathscr{B}(y \circ x, z) \\
& =\mathscr{B}(y, x \circ z)=\left\langle\operatorname{rg}^{*}(x) \varphi(y), z\right\rangle, \quad \forall x, y, z \in J .
\end{aligned}
$$

That is, the representations $(\mathrm{rg}, J)$ and $\left(\mathrm{rg}^{*}, J^{*}\right)$ are isomorphic. Let $a^{*}=$ $\varphi(x), b^{*}=\varphi(y)$, then by Proposition 3.1, $r$ is a solution of JYBE in $J$ if and only if

$$
\begin{aligned}
r \varphi(x) \circ r \varphi(y)=r\left(a^{*}\right) \circ r\left(b^{*}\right) & =r\left(\operatorname{rg}^{*}\left(r\left(a^{*}\right)\right) b^{*}+\operatorname{rg}^{*}\left(r\left(b^{*}\right)\right) a^{*}\right) \\
& =r \varphi(r \varphi(x) \circ y+x \circ r \varphi(y)) .
\end{aligned}
$$

Therefore the conclusion holds.

Definition 3.5. Let $(J, \circ)$ be a Jordan algebra and $\rho: J \rightarrow \operatorname{gl}(V)$ be a representation. A linear map $T: V \rightarrow J$ is called an $\mathcal{O}$-operator of $J$ associated to $\rho$ if $T$ satisfies

$$
T(u) \circ T(v)=T(\rho(T(u)) v+\rho(T(v)) u), \quad \forall u, v \in V .
$$

ExAmple 3.6. Let $(J, \circ)$ be a Jordan algebra. Then a Rota-Baxter operator (of weight zero) is an $\mathcal{O}$-operator of $J$ associated to the regular representation $(\mathrm{rg}, J)$ and a skew-symmetric solution of JYBE in $J$ is an $\mathcal{O}$-operator of $J$ associated to the representation $\left(\mathrm{rg}^{*}, J^{*}\right)$.

TheOREM 3.7. Let $(J, \circ)$ be a Jordan algebra. Let $\rho: J \rightarrow \operatorname{gl}(V)$ be a representation of $J$ and $\rho^{*}: J \rightarrow \mathrm{gl}\left(V^{*}\right)$ be the dual representation. Let $T: V \rightarrow J$ be a linear map which can be identified as an element in $\left(J \ltimes_{\rho^{*}} V^{*}\right) \otimes\left(J \ltimes_{\rho^{*}} V^{*}\right)$. Then $r=T-\sigma(T)$ is a skew-symmetric solution of JYBE in $J \ltimes_{\rho^{*}} V^{*}$ if and only if $T$ is an $\mathcal{O}$-operator of $J$ associated to $\rho$. 
Proof. Let $\left\{e_{1}, \ldots, e_{n}\right\}$ be a basis of $J$. Let $\left\{v_{1}, \ldots, v_{m}\right\}$ be a basis of $V$ and $\left\{v_{1}^{*}, \ldots, v_{m}^{*}\right\}$ be the dual basis. Set $T\left(v_{i}\right)=\sum_{k=1}^{n} a_{i k} e_{k}, i=1, \ldots, m$. Then, we have

$$
T=\sum_{i=1}^{m} T\left(v_{i}\right) \otimes v_{i}^{*}=\sum_{i=1}^{m} \sum_{k=1}^{n} a_{i k} e_{k} \otimes v_{i}^{*} \in J \otimes V^{*} \subset\left(J \ltimes_{\rho^{*}} V^{*}\right) \otimes\left(J \ltimes_{\rho^{*}} V^{*}\right) .
$$

Therefore we have

$$
\begin{aligned}
r_{12} \circ r_{13}= & \sum_{i, k=1}^{m}\left\{T\left(v_{i}\right) \circ T\left(v_{k}\right) \otimes v_{i}^{*} \otimes v_{k}^{*}-\rho^{*}\left(T\left(v_{i}\right)\right) v_{k}^{*} \otimes v_{i} \otimes T\left(v_{k}\right)\right. \\
& \left.-\rho^{*}\left(T\left(v_{k}\right)\right) v_{i}^{*} \otimes T\left(v_{i}\right) \otimes v_{k}^{*}\right\} ; \\
r_{12} \circ r_{23}= & \sum_{i, j=1}^{m}\left\{-v_{i}^{*} \otimes T\left(v_{i}\right) \circ T\left(v_{k}\right) \otimes v_{k}^{*}+T\left(v_{i}\right) \otimes \rho^{*}\left(T\left(v_{k}\right)\right) v_{i}^{*} \otimes v_{k}^{*}\right. \\
& \left.+v_{i}^{*} \otimes \rho^{*}\left(T\left(v_{i}\right)\right) v_{k}^{*} \otimes T\left(v_{k}\right)\right\} ; \\
r_{13} \circ r_{23}= & \sum_{i, k=1}^{m}\left\{v_{i}^{*} \otimes v_{k}^{*} \otimes T\left(v_{i}\right) \circ T\left(v_{k}\right)-T\left(v_{i}\right) \otimes v_{k}^{*} \otimes \rho^{*}\left(T\left(v_{k}\right)\right) v_{i}^{*}\right. \\
& \left.\quad-v_{i}^{*} \otimes T\left(v_{k}\right) \otimes \rho^{*}\left(T\left(v_{i}\right)\right) v_{k}^{*}\right\} .
\end{aligned}
$$

By the definition of dual representation, we know

$$
\rho^{*}\left(T\left(v_{k}\right)\right) v_{i}^{*}=\sum_{j=1}^{m}\left\langle v_{i}^{*}, \rho\left(T\left(v_{k}\right)\right) v_{j}\right\rangle v_{j}^{*},
$$

Then

$$
\begin{array}{rl}
\sum_{i, k=1}^{m} & T\left(v_{i}\right) \otimes \rho^{*}\left(T\left(v_{k}\right)\right) v_{i}^{*} \otimes v_{k}^{*} \\
& =\sum_{i, k=1}^{m} \sum_{j=1}^{m}\left\langle v_{j}^{*}, \rho\left(T\left(v_{k}\right)\right) v_{i}\right\rangle T\left(v_{j}\right) \otimes v_{i}^{*} \otimes v_{k}^{*} \\
& =\sum_{i, k=1}^{m} T\left\{\left\langle v_{j}^{*}, \rho\left(T\left(v_{k}\right)\right) v_{i}\right\rangle v_{j}\right\} \otimes v_{i}^{*} \otimes v_{k}^{*} \\
& =\sum_{i, k=1}^{m} T\left\{\rho\left[T\left(v_{k}\right)\right] v_{i}\right\} \otimes v_{i}^{*} \otimes v_{k}^{*} .
\end{array}
$$


Hence, we get

$$
\begin{aligned}
& r_{12} \circ r_{13}+r_{13} \circ r_{23}-r_{12} \circ r_{23} \\
&= \sum_{i, k=1}^{m}\left\{\left\{T\left(v_{i}\right) \circ T\left(v_{k}\right)-\rho\left(T\left(v_{i}\right)\right) v_{k}-\rho\left(T\left(v_{k}\right)\right) v_{i}\right\} \otimes v_{i}^{*} \otimes v_{k}^{*}\right. \\
& \quad+v_{i}^{*} \otimes\left\{T\left(v_{i}\right) \circ T\left(v_{k}\right)-\rho\left(T\left(v_{i}\right)\right) v_{k}-\rho\left(T\left(v_{k}\right)\right) v_{i}\right\} \otimes v_{k}^{*} \\
&\left.\quad+v_{i}^{*} \otimes v_{k}^{*} \otimes\left\{T\left(v_{i}\right) \circ T\left(v_{k}\right)-\rho\left(T\left(v_{i}\right)\right) v_{k}-\rho\left(T\left(v_{k}\right)\right) v_{i}\right\}\right\} .
\end{aligned}
$$

So $r$ is a solution of JYBE in $J \ltimes_{\rho^{*}} V^{*}$ if and only if $T$ is an $\mathcal{O}$-operator of $J$ associated to $\rho$.

Combining Proposition 3.1 and Theorem 3.7, we have the following conclusion.

Corollary 3.8. Let $(J, \circ)$ be a Jordan algebra and $\rho: J \rightarrow \operatorname{gl}(V)$ be a representation. Set $\hat{J}=J \ltimes_{\rho^{*}} V^{*}$. Let $T: V \rightarrow J$ be a linear map. Then the following conditions are equivalent:

(1) $T$ is an $\mathcal{O}$-operator of $J$ associated to $\rho$.

(2) $T-\sigma(T)$ is a skew-symmetric solution of the JYBE in the Jordan algebra $\hat{J}$.

(3) $T-\sigma(T)$ is an $\mathcal{O}$-operator of the Jordan algebra $\hat{J}$ associated to $\left(\operatorname{rg}_{\hat{J}}^{*}, \hat{J}^{*}\right)$.

REMARK 3.9. The equivalence between the above (1) and (3) can be obtained by a straightforward proof and then Theorem 3.7 follows from this equivalence and Proposition 3.1.

\section{Pre-Jordan algebras}

\subsection{Definition and some basic properties}

Definition 4.1. A pre-Jordan algebra $A$ is a vector space equipped with a binary operation $(x, y) \rightarrow x \cdot y$ satisfying the following equations (for any $x, y, z, u \in A)$ :

$$
\begin{aligned}
& (x \circ y) \cdot(z \cdot u)+(y \circ z) \cdot(x \cdot u)+(z \circ x) \cdot(y \cdot u) \\
& =z \cdot[(x \circ y) \cdot u]+x \cdot[(y \circ z) \cdot u]+y \cdot[(z \circ x) \cdot u], \\
& x \cdot[y \cdot(z \cdot u)]+z \cdot[y \cdot(x \cdot u)]+[(x \circ z) \circ y] \cdot u \\
& =z \cdot[(x \circ y) \cdot u]+x \cdot[(y \circ z) \cdot u]+y \cdot[(z \circ x) \cdot u],
\end{aligned}
$$

where $x \circ y=x \cdot y+y \cdot x$. 
REMARK 4.2. Eqs. (4.1) and (4.2) are equivalent to the following equations (for any $x, y, z, u \in A$ ) respectively

$$
\begin{aligned}
& (x, y, z, u)_{1}+(y, z, x, u)_{1}+(z, x, y, u)_{1} \\
& \quad+(y, x, z, u)_{1}+(x, z, y, u)_{1}+(z, y, x, u)_{1}=0 \\
& (x, y, z \cdot u)-(x \cdot z, y, u)+(y, z, x, u)_{2} \\
& \quad+(y, x, z, u)_{2}+(z, y, x \cdot u)-(z \cdot x, y, u)=0
\end{aligned}
$$

where $(x, y, z)=(x \cdot y) \cdot z-x \cdot(y \cdot z)$ is the associator and

$$
\begin{aligned}
& (x, y, z, u)_{1}=(x \cdot y) \cdot(z \cdot u)-x \cdot[(y \cdot z) \cdot u], \\
& (x, y, z, u)_{2}=(x \cdot y) \cdot(z \cdot u)-[x \cdot(y \cdot z)] \cdot u .
\end{aligned}
$$

COROLlaRy 4.3. Any associative algebra is a pre-Jordan algebra.

Proof. It follows immediately from Eqs. (4.3) and (4.4).

Proposition 4.4. Let $(A, \cdot)$ be a pre-Jordan algebra. Then the operation given by

$$
x \circ y=x \cdot y+y \cdot x, \quad \forall x, y \in A,
$$

defines a Jordan algebra $J(A)$, which is called the associated Jordan algebra of $(A, \cdot)$ and $(A, \cdot)$ is also called a compatible pre-Jordan algebra structure on the Jordan algebra $(J(A), \circ)$.

Proof. For any $x, y, z, a \in A$, it is easy to show that

$$
\begin{aligned}
(x \circ y) \circ(a \circ z) & +(y \circ z) \circ(a \circ x)+(z \circ x) \circ(a \circ y) \\
& =[(x \circ y) \circ a] \circ z+[(y \circ z) \circ a] \circ x+[(z \circ x) \circ a] \circ y
\end{aligned}
$$

if and only if $l_{1}+l_{2}+l_{3}=r_{1}+r_{2}+r_{3}$, where

$$
\begin{aligned}
l_{1}= & (x \circ y) \cdot(a \cdot z)+(y \circ a) \cdot(x \cdot z)+(x \circ a) \cdot(y \cdot z), \\
l_{2}= & (x \circ a) \cdot(z \cdot y)+(z \circ a) \cdot(x \cdot y)+(x \circ z) \cdot(a \cdot y), \\
l_{3}= & (a \circ y) \cdot(z \cdot x)+(y \circ z) \cdot(a \cdot x)+(z \circ a) \cdot(y \cdot x), \\
r_{1}= & {[(x \cdot y) \cdot a] \cdot z+x \cdot[a \cdot(y \cdot z)]+[(y \cdot x) \cdot a] \cdot z } \\
& \quad+y \cdot[a \cdot(x \cdot z)]+[a \cdot(x \cdot y)] \cdot z+[a \cdot(y \cdot x)] \cdot z, \\
r_{2}= & x \cdot[a \cdot(z \cdot y)]+[(x \cdot z) \cdot a] \cdot y+[a \cdot(x \cdot z)] \cdot y \\
& \quad+[a \cdot(z \cdot x)] \cdot y+z \cdot[a \cdot(x \cdot y)]+[(z \cdot x) \cdot a] \cdot y,
\end{aligned}
$$




$$
\begin{aligned}
r_{3}=[a \cdot(y \cdot z)] \cdot x+[a \cdot(z \cdot y)] \cdot x+y \cdot[a \cdot(z \cdot x)] \\
+[(y \cdot z) \cdot a] \cdot x+z \cdot[a \cdot(y \cdot x)]+[(z \cdot y) \cdot a] \cdot x .
\end{aligned}
$$

Note that

$$
\begin{aligned}
& r_{1}= {[(x \cdot y) \cdot a] \cdot z+x \cdot[a \cdot(y \cdot z)]+[(y \cdot x) \cdot a] \cdot z+y \cdot[a \cdot(x \cdot z)] } \\
&+[a \cdot(x \cdot y)] \cdot z+[a \cdot(y \cdot x)] \cdot z \\
&=(x \cdot y) \cdot(a \cdot z)+(x \cdot a) \cdot(y \cdot z)+(y \cdot x) \cdot(a \cdot z)+(y \cdot a) \cdot(x \cdot z) \\
& \quad+(a \cdot x) \cdot(y \cdot z)+(a \cdot y) \cdot(x \cdot z)=l_{1} .
\end{aligned}
$$

Similarly, we have $l_{2}=r_{2}, l_{3}=r_{3}$.

The identities (4.1) and (4.2) are given from the following conclusion:

PROPOSITION 4.5. Let $A$ be a vector space with a binary operation denoted by $\cdot$. Then $(A, \cdot)$ is a pre-Jordan algebra if and only if $(A, \circ)$ defined by Eq. (4.6) is a Jordan algebra and $(L ., A)$ is a representation of $(A, \circ)$.

PROOF. “ $\Rightarrow$ " It is exactly Proposition 4.4.

" $\Leftarrow$ " It can be obtained straightforwardly or by a similar proof as of Proposition 4.6.

\subsection{Pre-Jordan algebras and $\mathcal{O}$-operators of Jordan algebras}

Proposition 4.6. Let $(J, \circ)$ be a Jordan algebra and $(\rho, V)$ be a representation. If $T$ is an $\mathscr{O}$-operator associated to $(\rho, V)$, then there exists a pre-Jordan algebra structure on $V$ defined by

$$
u * v=\rho(T(u)) v, \quad \forall u, v \in V .
$$

Therefore there exists an associated Jordan algebra structure on $V$ given by Eq. (4.6) and $T$ is a homomorphism of Jordan algebras. Moreover, $T(V)=$ $\{T(v) \mid v \in V\} \subset J$ is a Jordan subalgebra of $(J, \circ)$ and there is an induced pre-Jordan algebra structure on $T(V)$ given by

$$
T(u) \cdot T(v)=T(u * v), \quad \forall u, v \in V .
$$

The corresponding associated Jordan algebra structure on $T(V)$ given by $E q$. (4.6) is just a Jordan subalgebra of $(J, \circ)$ and $T$ is a homomorphism of pre-Jordan algebras. 
Proof. For any $u, v, w, a \in V$, we set $x=T(u), y=T(v), z=T(w)$ and $u \circ v=u * v+v * u$.

$$
\begin{aligned}
(u \circ v) *(w * a) & =[\rho(T(u)) v+\rho(T(v)) u] *(\rho(T(w)) a) \\
& =\rho\{T[\rho(T(u)) v+\rho(T(v)) u]\} \rho(T(w)) a \\
& =\rho[T(u) \circ T(v)] \rho(T(w)) a=\rho(x \circ y) \rho(z) a, \\
u *[(v \circ w) * a] & =\rho(T(u)) \rho\{T[\rho(T(v)) w+\rho(T(w)) v]\} a \\
& =\rho(T(u)) \rho[T(v) \circ T(w)] a=\rho(x) \rho(y \circ z) a, \\
u *[v *(w * a)] & =\rho(T(u)) \rho(T(v)) \rho(T(w)) a=\rho(x) \rho(y) \rho(z) a, \\
{[(u \circ w) \circ v] * a } & =\rho\{T[\rho(T(u \circ w)) v+\rho(T(v))(u \circ w)]\} a \\
& =\rho\{T(u \circ w) \circ T(v)\} a \\
& =\rho\{T[\rho(T(u)) w+\rho(T(w)) u] \circ T(v)\} a \\
& =\rho\{(T(u) \circ T(w)) \circ T(v)\} a=\rho((x \circ z) \circ y) a .
\end{aligned}
$$

Hence

$$
\begin{aligned}
(u \circ v) & *(w * a)+(v \circ w) *(u * a)+(w \circ u) *(v * a) \\
& =\rho(x \circ y) \rho(z) a+\rho(y \circ z) \rho(x) a+\rho(z \circ x) \rho(y) a \\
& =\rho(z) \rho(x \circ y) a+\rho(x) \rho(y \circ z) a+\rho(y) \rho(z \circ x) a \\
& =w *[(u \circ v) * a]+u *[(v \circ w) * a]+v *[(w \circ u) * a], \\
u *[v & *(w * a)]+w *[v *(u * a)]+[(u \circ w) \circ v] * a \\
& =\rho(x) \rho(y) \rho(z) a+\rho(z) \rho(y) \rho(x) a+\rho((x \circ z) \circ y) a \\
& =\rho(z) \rho(x \circ y) a+\rho(x) \rho(y \circ z) a+\rho(y) \rho(z \circ x) a \\
& =w *[(u \circ v) * a]+u *[(v \circ w) * a]+v *[(w \circ u) * a] .
\end{aligned}
$$

So $(V, *)$ is a pre-Jordan algebra. The other conclusions follow immediately.

A direct consequence of Proposition 4.6 is the following construction of a pre-Jordan algebra from a Rota-Baxter operator (of weight zero) of a Jordan algebra.

COROllary 4.7. Let $(J, \circ)$ be a Jordan algebra and $R$ be a Rota-Baxter operator (of weight zero) on J. Then there is a pre-Jordan algebra structure 
on J given by

$$
x \cdot y=R(x) \circ y, \quad \forall x, y \in J
$$

Proof. It follows immediately from Example 3.6 and Proposition 4.6.

COROLlary 4.8. Let $(J, \circ)$ be a Jordan algebra. Then there exists a compatible pre-Jordan algebra structure on $J$ if and only if there exists an invertible $\mathcal{O}$-operator of $(\mathrm{J}, \mathrm{O})$.

Proof. If there exists an invertible $\mathscr{O}$-operator of $(J, \circ)$ associated to a representation $(\rho, V)$, then by Proposition 4.6, there is a pre-Jordan algebra structure on $J$ given by

$$
x \cdot y=T\left(\rho(x) T^{-1}(y)\right), \quad \forall x, y \in J .
$$

It is a compatible pre-Jordan algebra structure on $(J . \circ)$ since

$$
\begin{aligned}
x \cdot y+y \cdot x & =T\left(\rho(x) T^{-1}(y)\right)+T\left(\rho(y) T^{-1}(x)\right) \\
& =T\left(T^{-1}(x) \circ T^{-1}(y)\right)=x \circ y, \quad \forall x, y \in J .
\end{aligned}
$$

Conversely, let $(J, \cdot)$ be a pre-Jordan algebra and $(J, \circ)$ be the associated Jordan algebra. Then the identity map id $: A \rightarrow A$ is an $\mathscr{O}$-operator of $(J, \circ)$ associated to $(L ., A)$.

The following conclusion reveals the relationship between pre-Jordan algebras and the Jordan algebras with a nondegenerate symplectic form:

Proposition 4.9. Let $(J, \circ)$ be a Jordan algebra with a nondegenerate symplectic form $\omega$. Then there exists a compatible pre-Jordan algebra structure “.” on J given by

$$
\omega(x \cdot y, z)=\omega(y, x \circ z), \quad \forall x, y \in J .
$$

Proof. Define a linear map $T: J \rightarrow J^{*}$ by $\langle T(x), y\rangle=\omega(x, y), \forall x, y \in$ $J$. Then $T$ is invertible and $T^{-1}$ is an $\mathscr{O}$-operator of $J$ associated to the representation $\left(\mathrm{rg}^{*}, J^{*}\right)$ since (for any $x, y, z \in J$ )

$$
\begin{aligned}
\langle T(x \circ y), z\rangle & =\omega(x \circ y, z)=\omega(y, x \circ z)+\omega(x, y \circ z) \\
& =\left\langle\operatorname{rg}^{*}(x) T(y), z\right\rangle+\left\langle\operatorname{rg}^{*}(y) T(x), z\right\rangle .
\end{aligned}
$$

By Corollary 4.8, there is a compatible pre-Jordan algebra structure "." on $(J, \circ)$ given by

$$
x \cdot y=T^{-1}\left(\operatorname{rg}^{*}(x) T(y)\right), \quad \forall x, y \in J,
$$


which gives exactly

$$
\begin{aligned}
\omega(x \cdot y, z) & =\langle T(x \cdot y), z\rangle=\left\langle\operatorname{rg}^{*}(x) T(y), z\right\rangle \\
& =\langle T(y), x \circ z\rangle=\omega(y, x \circ z), \quad \forall x, y, z \in J .
\end{aligned}
$$

So the conclusion holds.

The following conclusion provides a construction of solutions of JYBE in certain Jordan algebras from pre-Jordan algebras.

Corollary 4.10. Let $(A, \cdot)$ be a pre-Jordan algebra. Then

$$
r=\sum_{i}^{n}\left(e_{i} \otimes e_{i}^{*}-e_{i}^{*} \otimes e_{i}\right)
$$

is a skew-symmetric solution of Jordan Yang-Baxter equation in the Jordan algebra $J(A) \ltimes_{L^{*}} J(A)^{*}$, where $\left\{e_{1}, \ldots, e_{n}\right\}$ is a basis of $A$ and $\left\{e_{1}^{*}, \ldots, e_{n}^{*}\right\}$ is the dual basis. Moreover, there is a symplectic form $\omega$ on $J(A) \ltimes_{L^{*}} J(A)^{*}$ induced by $r$ through Eq. (1.10) which is given by Eq. (1.12).

Proof. Since id is an $\mathscr{O}$-operator of the associated Jordan algebra $(J(A), \circ)$ associated to $(L ., A)$, the conclusion follows from Theorem 3.7 and Proposition 2.12.

\subsection{Relationships with dendriform algebras}

Definition 4.11 ([39]). A dendriform algebra $(A, \succ, \prec)$ is a vector space $A$ equipped with two binary operations denoted by $\succ, \prec: A \otimes A \rightarrow A$ satisfying the following equations:

$$
\begin{aligned}
& x \succ(y \succ z)=(x * y) \succ z, \\
& (x \succ y) \prec z=x \succ(y \prec z), \\
& (x \prec y) \prec z=x \prec(y * z),
\end{aligned}
$$

where $x, y, z \in A$ and $x * y=x \succ y+x \prec y$.

Proposition 4.12. Let $(A, \succ, \prec)$ be a dendriform algebra. Then the operation given by

$$
x \cdot y=x \succ y+y \prec x, \quad \forall x, y \in A,
$$

defines a pre-Jordan algebra structure on A.

Proof. For any $x, y \in A$, set $x * y=x \succ y+x \prec y$ and $x \circ y=x \cdot y+y \cdot x$. We divide the left hand side of Eq. (4.1) into three parts:

$$
(x \circ y) \cdot(z \cdot u)+(y \circ z) \cdot(x \cdot u)+(z \circ x) \cdot(y \cdot u)=T_{1}+T_{2}+T_{3}, \quad \forall x, y, z, u \in A,
$$


where

$$
\begin{aligned}
T_{1}=[ & (x * y) * z] \succ u+u \prec[z *(y * x)]+(x \succ u) \prec(y * z) \\
& +(y * z) \succ(u \prec x)+(x \succ u) \prec(z * y)+(z * y) \succ(u \prec x) \\
& +u \prec[y *(z * x)]+[(x * z) * y] \succ u, \\
T_{2}=u & \prec[z *(x * y)]+[(y * x) * z] \succ u+[(y * z) * x] \succ u \\
& +u \prec[x *(z * y)]+(y \succ u) \prec(z * x)+(z * x) \succ(u \prec y) \\
& +(y \succ u) \prec(x * z)+(x * z) \succ(u \prec y), \\
T_{3}=(z & \succ u) \prec(x * y)+(x * y) \succ(u \prec y)+(z \succ u) \prec(y * x) \\
& +(y * x) \succ(u \prec z)+u \prec[x *(y * z)]+[(z * y) * x] \succ u \\
& +[(z * x) * y] \succ u+u \prec[y *(z * x)] .
\end{aligned}
$$

Note that

$$
\begin{aligned}
T_{1}=x & \succ[(y * z) \succ u]+[(y * z) \succ u] \prec x+x \succ[u \prec(y * z)] \\
& +[u \prec(y * z)] \prec x+x \succ[(z * y) \succ u]+[(z * y) \succ u] \prec x \\
& +x \succ[u \prec(z * y)]+[u \prec(z * y)] \prec x \\
=x & {[(y \circ z) \cdot u], } \\
T_{2}=y & \succ[(z * x) \succ u]+[(z * x) \succ u] \prec y+y \succ[u \prec(z * x)] \\
& +[u \prec(z * x)] \prec y+y \succ[(x * z) \succ u]+[(x * z) \succ u] \prec y \\
& +y \succ[u \prec(x * z)]+[u \prec(x * z)] \prec y \\
=y & \cdot[(z \circ x) \cdot u], \\
T_{3}=z & \succ[(x * y) \succ u]+[(x * y) \succ u] \prec z+z \succ[u \prec(x * y)] \\
& +[u \prec(x * y)] \prec z+z \succ[(y * x) \succ u]+[(y * x) \succ u] \prec z \\
& +z \succ[u \prec(y * x)]+[u \prec(y * x)] \prec z \\
=z & \cdot[(x \circ y) \cdot u] .
\end{aligned}
$$

So Eq. (4.1) holds. Similarly, Eq. (4.2) holds.

The following conclusion is obvious.

Corollary 4.13. Let $(A, \succ, \prec)$ be a dendriform algebra. Then the operation given by

$$
\begin{aligned}
x \circ y & =x * y+y * x=x \cdot y+y \cdot x \\
& =x \succ y+y \prec x+x \prec y+y \succ x, \quad \forall x, y \in A,
\end{aligned}
$$

defines a Jordan algebra structure on $A$, where $(A, *)$ is the associative algebra defined by $\mathrm{Eq} .(1.2)$ and $(A, \cdot)$ is the pre-Jordan algebra defined by 
Eq. (4.13). That is, the Jordan algebras as the anticommutator of $(A, *)$ and $(A, \cdot)$ respectively coincide.

Therefore we have the commutative diagram (1.5). Moreover, combining it with diagram (1.1), we have the following (detailed) commutative diagram:

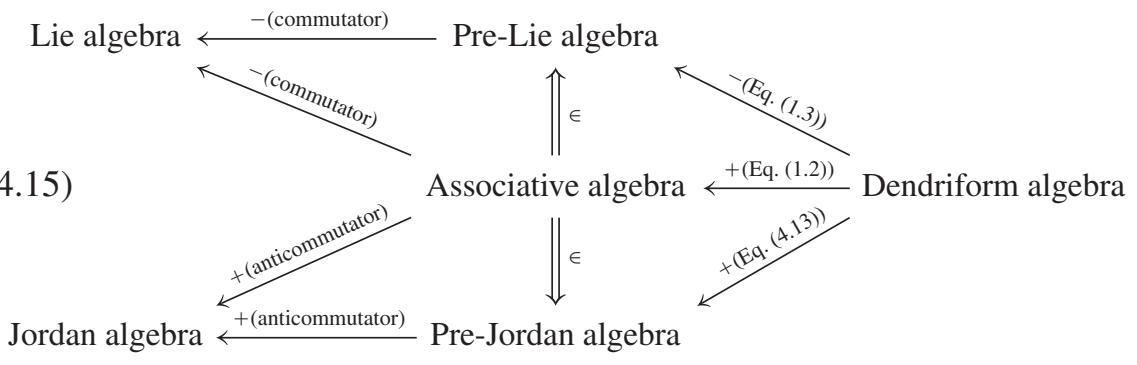

where both " $\uparrow \epsilon$ " and " $\downarrow \in$ " mean the inclusion.

\section{5. $\mathscr{O}$-operators of pre-Jordan algebras}

\subsection{Bimodules of pre-Jordan algebras}

Definition 5.1. Let $(A, \cdot)$ be a pre-Jordan algebra and $V$ be a vector space. Let $l ., r .: A \rightarrow \operatorname{gl}(V)$ be two linear maps. Then $V$ (or the pair $(l ., r$.), or $(l, r, V))$ is called a bimodule of $A$ if the following conditions hold (for any $x, y, z \in A)$ :

$$
\begin{aligned}
& {[l .(x \circ y), l .(z)]+[l .(y \circ z), l .(x)]+[l .(z \circ x), l .(y)]=0,} \\
& l .(x \circ y) r .(z)+r(x \cdot z) l .(y)+r .(y \cdot z) r .(x) \\
& +r .(x \cdot z) r .(y)+r .(y \cdot z) l .(x)=l .(x) r .(z) l .(y)+l .(y) r .(z) r .(x) \\
& +r .[(x \circ y) z]+l .(y) r .(z) l .(x)+l .(x) r .(z) r .(y), \\
& l .(x \circ y) l .(z)+l .(y \circ z) l .(x)+l .(z \circ x) l .(y) \\
& =l .(x) l .(y) l .(z)+l .[y \circ(x \circ z)]+l .(z) l .(y) l .(x),
\end{aligned}
$$

$$
\begin{aligned}
& r .(z \cdot y) l .(x)+r .(x \cdot y) r .(z)+l .(x \circ z) r .(y) \\
& +r .(x \cdot y) l .(z)+r .(z \cdot y) r .(x)=l .(x) r .(z \cdot y)+r .(y) r .(x \circ z) \\
& +r .(y) l .(x \circ z)+l .(z) r .(x \cdot y), \\
& l .(x \cdot y) r .(z)+r .(x \cdot z) l .(y)+r .(y \cdot z) r .(x)+l .(y \cdot x) r .(z) \\
& +r .(x \cdot z) r .(y)+r .(y \cdot z) l .(x)=l .(x) l .(y) r .(z)+r .(z) l .(y) r .(x) \\
& +r .(z) r .(y) r .(x)+r .(z) l .(y) l .(x)+r .[y \cdot(x \cdot z)]+r .(z) r .(y) l(x) \text {, }
\end{aligned}
$$

where $x \circ y=x \cdot y+y \cdot x$. 
According to [46], $(l, r, r)$ is a bimodule of a pre-Jordan algebra $(A, \cdot)$ if and only if the direct sum $A \oplus V$ (as vector space) turns into a pre-Jordan algebra (the semidirect sum) by defining multiplication in $A \oplus V$ by

$$
(x+u) *(y+v)=x \cdot y+(l .(x) v+r .(y) u), \quad \forall x, y \in A, u, v \in V .
$$

We denote it by $A \ltimes_{l, r}, V$ or simply $A \ltimes V$.

Proposition 5.2. Let $(l ., r$. be a bimodule of a pre-Jordan algebra $(A, \cdot)$. Let $J(A)$ be the associated Jordan algebra.

(1) $(l, V)$ is a representation of $J(A)$.

(2) $(l .+r, V)$ is a representation of $J(A)$.

(3) If $\rho: J(A) \rightarrow \mathrm{gl}(V)$ is a representation of $J(A)$, then $(\rho, 0, V)$ is a bimodule of $(A, \cdot)$.

(4) Both $(l ., 0, V)$ and $(l .+r ., 0, V)$ are bimodules of $(A, \cdot)$.

(5) The pre-Jordan algebras $A \ltimes_{l, r}, V$ and $A \ltimes_{l,+r, 0} V$ have the same associated Jordan algebra $J(A) \ltimes_{l,+r}$. $V$.

Proof. (1) and (3) follows directly from Eqs. (5.1) and (5.3). Moreover, $(l, r, V)$ is a bimodule of a pre-Jordan algebra $(A, \cdot)$ if and only if $(l .+r ., V)$ is a representation of the associated Jordan algebra $(J(A), \circ)$ and Eqs. (5.2), (5.4) and (5.5) hold. Thus (2) holds. (4) follows from (1), (2) and (3). (5) follows from the relationship between a pre-Jordan algebra and the associated Jordan algebra.

Proposition-Definition 5.3. Let $(A, \cdot)$ be a pre-Jordan algebra and $(l$, $r, V)$ be a bimodule. Then $\left(l^{*}+r_{*}^{*},-r_{.}^{*}, V^{*}\right)$ is a bimodule of $(A, \cdot)$. It is called the dual bimodule of $(l, r, r)$.

Proof. This conclusion can be obtained by a straightforward proof on the Eqs. (5.1)-(5.5). We give another approach by using the relationships between the bimodules of a pre-Jordan algebra $(A, \cdot)$ and the representations of the associated Jordan algebra $(J(A), \circ)$ with the (known) dual representations of $(J(A), \circ)$. Let $\left(\bar{l}_{.}, \bar{r}_{\mathrm{r}}, V^{*}\right)$ be the dual bimodule of $(l, r, r)$. Then by Proposition 5.2 , both $\left(\bar{l} .+\bar{r}, V^{*}\right)$ and $\left(\bar{l} ., V^{*}\right)$ are representations of the Jordan algebra $(J(A), \circ)$. On the other hand, since both $(l .+r ., V)$ and $(l ., V)$ are representations of the Jordan algebra $(J(A), \circ)$, by Proposition-Definition 2.5, their dual bimodules are $\left(l_{.}^{*}+r_{.}^{*}, V^{*}\right)$ and $\left(l_{.}^{*}, V^{*}\right)$ respectively. Therefore we have the following equations

$$
\bar{l} .+\bar{r} .=l^{*}, \quad \bar{l} .=l^{*}+r^{*} .
$$

Thus $\left(l^{*}+r^{*},-r_{.}^{*}, V^{*}\right)$ is a bimodule of $(A, \cdot)$. 
By Propositions 5.2. and Proposition-Definition 5.3, the following conclusion is obvious.

Corollary 5.4. Let $(A, \cdot)$ be a pre-Jordan algebra and $(l ., r ., V)$ be a bimodule.

(1) Both $\left(l_{.}^{*}+r_{.}^{*}, 0, V^{*}\right)$ and $\left(l_{.}^{*}, 0, V^{*}\right)$ are bimodules of $(A, \cdot)$.

(2) Both $\left(l^{*}+r_{.}^{*}, V^{*}\right)$ and $\left(l_{.}^{*}, V^{*}\right)$ are representations of the associated Jordan algebra $J(A)$.

(3) The pre-Jordan algebras $A \ltimes_{l_{*}^{*}, 0} V^{*}$ and $A \ltimes_{l_{*}^{*}+r_{*}^{*},-r_{*}^{*}} V^{*}$ have the same associated Jordan algebra $J(A) \ltimes_{l^{*}} V^{*}$.

Proposition-Definition 5.5. Let $(A, \cdot)$ be a pre-Jordan algebra. Then $(L ., R ., A),(L ., 0, A)$ and $(\mathrm{rg}, 0, A)$ are bimodules of $A$ and the first one is called the regular bimodule of $A$, where $\operatorname{rg}=L .+R$. On the other hand, $\left(\mathrm{rg}^{*},-R_{.}^{*}, A^{*}\right),\left(L_{.}^{*}, 0, A^{*}\right)$, and $\left(\mathrm{rg}^{*}, 0, A^{*}\right)$ are bimodules of $A$ too.

\section{2. $\mathcal{O}$-operators of pre-Jordan algebras and $J P$-equation}

Definition 5.6. Let $(A, \cdot)$ be a pre-Jordan algebra and $(l, r ., V)$ be a bimodule. A linear map $T: V \rightarrow A$ is called an $\mathcal{O}$-operator of $A$ associated to $(l, r, V)$ if

$$
T(u) \cdot T(v)=T(l \cdot(T(u)) v+r .(T(v)) u), \quad \forall u, v \in V .
$$

In particular, an $\mathcal{O}$-operator of a pre-Jordan algebra $(A, \cdot)$ associated to the regular bimodule $(L ., R ., A)$ is called a Rota-Baxter operator (of weight zero) on $(A, \cdot)$.

The following conclusion is obvious.

COROLlary 5.7. Let $T$ be an $\mathcal{O}$-operator of a pre-Jordan algebra $(A, \cdot)$ associated to a bimodule $(l ., r, V)$. Then $T$ is an $\mathcal{O}$-operator of the associated Jordan algebra $J(A)$ associated to $(l .+r ., V)$.

Proposition 5.8. Let $(A, \cdot)$ be a pre-Jordan algebra and $r \in A \otimes A$ be symmetric. Then the following conditions are equivalent.

(1) $r$ is an $\mathcal{O}$-operator of the associated Jordan algebra $(J(A), \circ)$ associated to $\left(L_{.}^{*}, A^{*}\right)$.

(2) $r$ is an $\mathcal{O}$-operator of $(A, \cdot)$ associated to $\left(\mathrm{rg}^{*},-R^{*}, A^{*}\right)$.

(3) $r$ satisfies

$$
r_{13} \circ r_{23}-r_{12} \cdot r_{23}-r_{12} \cdot r_{13}=0 .
$$


Proof. Let $\left\{e_{1}, \ldots, e_{n}\right\}$ be a basis of $A$ and $\left\{e_{1}^{*}, \ldots, e_{n}^{*}\right\}$ be the dual basis. Suppose that $e_{i} \cdot e_{j}=\sum_{k=1}^{n} a_{i j}^{k} e_{k}$ and $r=\sum_{i, j=1}^{n} r_{i j} e_{i} \otimes e_{j}, r_{i j}=r_{j i}$. Hence $r\left(e_{i}^{*}\right)=\sum_{k=1}^{n} r_{i k} e_{k}$. Then $r$ satisfies Eq. (5.8) if and only if (for any $i, j, t$ )

$$
\sum_{k, l=1}^{n}\left\{r_{i k} r_{j l}\left(a_{k l}^{t}+a_{l k}^{t}\right)-r_{i k} r_{l t} a_{k l}^{j}-r_{l j} r_{k t} a_{l k}^{i}\right\}=0 .
$$

The left-hand side of the above equation is just the coefficient of $e_{t}$ and $e_{j}$ in

$$
r\left(e_{i}^{*}\right) \circ r\left(e_{j}^{*}\right)-r\left(L_{.}^{*}\left(r\left(e_{i}^{*}\right)\right) e_{j}^{*}+L_{.}^{*}\left(r\left(e_{j}^{*}\right)\right) e_{i}^{*}\right)
$$

and

$$
r\left(\operatorname{rg}^{*}\left(r\left(e_{i}^{*}\right)\right) e_{t}^{*}\right)-r\left(e_{i}^{*}\right) \cdot r\left(e_{t}^{*}\right)-r\left(R_{.}^{*}\left(r\left(e_{t}^{*}\right)\right) e_{i}^{*}\right)
$$

respectively. Hence the conclusion holds.

Definition 5.9. Let $(A, \cdot)$ be a pre-Jordan algebra and $r \in A \otimes A$. Eq. (5.8) is called $J P$-equation in $A$.

Remark 5.10. Like the CYBE in a Lie algebra ([36]) and the JYBE in a Jordan algebra (see Proposition 3.1), the $J P$-equation in a pre-Jordan algebra can be regarded as an analogue of the CYBE (and the JYBE) in the sense of Proposition 5.8. Furthermore, like the close relationship between the JYBE and the Jordan D-bialgebras given in Proposition 2.10, in a subsequent work [29], we shall show that $J P$-equation arises naturally in the study of certain bialgebra structure on a pre-Jordan algebra.

Theorem 5.11. Let $(A, \cdot)$ be a pre-Jordan algebra. Let $(l, r, r)$ be a bimodule of $(A, \cdot)$ and $\left(l_{*}^{*}+r^{*},-r^{*}, V^{*}\right)$ be the dual bimodule. Let $T: V \rightarrow J$ be a linear map which can be identified as an element in $\left(A \ltimes_{\left.l_{*}^{*}+r_{*}^{*},-r_{*}^{*} V^{*}\right) \otimes}\right.$ $\left(A \ltimes_{l_{*}^{*}+r_{*}^{*},-r_{*}^{*}} V^{*}\right)$. Then $r=T+\sigma(T)$ is a symmetric solution of $J P$-equation in the pre-Jordan algebra $A \ltimes_{l_{*}^{*}+r_{*}^{*},-r_{*}^{*}} V^{*}$ if and only if $T$ is an $\mathcal{O}$-operator of $(A, \cdot)$ associated to $(l, r, r)$.

Proof. This conclusion follows from a similar proof as of Theorem 3.7.

Corollary 5.12. Let $(J, \circ)$ be a Jordan algebra and $(\rho, V)$ be a representation. Suppose that $T: V \rightarrow J$ is an $\mathcal{O}$-operator of $(J, \circ)$ associated to $(\rho, V)$. Then $r=T+\sigma(T)$ is a symmetric solution of $J P$-equation in the pre-Jordan algebra $T(V) \ltimes_{\rho^{*}, 0} V^{*}$, where $T(V) \subset A$ is a pre-Jordan algebra given by Eq. (4.8) and $\left(\rho^{*}, 0, V^{*}\right)$ is a bimodule of $T(V)$ since the associated Jordan algebra is a Jordan subalgebra of $(J, \circ)$ and $T$ can be identified as an element in $T(V) \otimes V^{*} \subset\left(T(V) \ltimes_{\rho^{*}, 0} V^{*}\right) \otimes\left(T(V) \ltimes_{\rho^{*}, 0} V^{*}\right)$. 
Proof. The conclusion follows immediately from Theorem 5.11 since $T$ is an $\mathscr{O}$-operator of the pre-Jordan algebra $T(V)$ associated to the bimodule $(\rho, 0, V)$.

REMARK 5.13. By Theorem 3.7 and Corollary 5.12, roughly speaking, the symmetric part of an $\mathscr{O}$-operator of a Jordan algebra corresponds to a symmetric solution of $J P$-equation, whereas the skew-symmetric part of an $\mathscr{O}$-operator of a Jordan algebra corresponds to a skew-symmetric solution of JYBE.

\subsection{Bilinear forms on pre-Jordan algebras and JP-equation}

Proposition 5.14. Let $(A, \cdot)$ be a pre-Jordan algebra and $T: A^{*} \rightarrow A$ be an invertible linear map.

(1) $T$ is an $\mathcal{O}$-operator of $(A, \cdot)$ associated to the bimodule $\left(L^{*}+R^{*}, 0, A^{*}\right)$ if and only if the bilinear form $\mathscr{B}$ induced by $T$ through Eq. (1.10) satisfies

$$
\mathscr{B}(x \cdot y, z)=\mathscr{B}(y, x \cdot z+z \cdot x), \quad \forall x, y \in A .
$$

(2) $T$ is an $\mathcal{O}$-operator of $(A, \cdot)$ associated to the bimodule $\left(L_{.}^{*}+R_{.}^{*}\right.$, $\left.-R_{.}^{*}, A^{*}\right)$ if and only if the bilinear form $\mathscr{B}$ induced by Through Eq. (1.10) satisfies

(5.10) $\mathscr{B}(x \cdot y, z)=\mathscr{B}(y, x \cdot z)+\mathscr{B}(y, z \cdot x)-\mathscr{B}(x, z \cdot y), \quad \forall x, y, z \in A$

(3) $T$ is an $\mathcal{O}$-operator of the associated Jordan algebra $(J(A)$, o) associated to the bimodule $\left(L^{*}, A^{*}\right)$ if and only if the bilinear form $\mathscr{B}$ induced by $T$ through Eq. (1.10) satisfies

$$
\mathscr{B}(x \circ y, z)=\mathscr{B}(y, x \cdot z)+\mathscr{B}(x, y \cdot z), \quad \forall x, y, z \in A .
$$

Proof. We only give an explicit proof of (1) and the proof of (2) and (3) is similar. For any $x, y, z \in A$, we set $x=T\left(a^{*}\right), y=T\left(b^{*}\right), z=T\left(c^{*}\right)$. Then we show that

$$
\begin{aligned}
\mathscr{B}(x & y, z)-\mathscr{B}(y, x \cdot z+z \cdot x) \\
& =\left\langle T^{-1}(x \cdot y), z\right\rangle-\left\langle T^{-1}(y), x \cdot z+z \cdot x\right\rangle \\
& =\left\langle T^{-1}\left(T\left(a^{*}\right) \cdot T\left(b^{*}\right)\right), z\right\rangle-\left\langle b^{*}, x \cdot z+z \cdot x\right\rangle \\
& =\left\langle T^{-1}\left(T\left(a^{*}\right) \cdot T\left(b^{*}\right)\right)-\left(L^{*}+R^{*}\right)(x) b^{*}, z\right\rangle \\
& =\left\langle T^{-1}\left(T\left(a^{*}\right) \cdot T\left(b^{*}\right)-T\left(L^{*}+R^{*}\right)(x) b^{*}\right), z\right\rangle .
\end{aligned}
$$

Hence (1) holds. 
Proposition 5.15. Let $(A, \cdot)$ be a pre-Jordan algebra and $\mathscr{B}$ be a bilinear form on A. If $\mathscr{B}$ satisfies Eq. (5.11), then

$$
\omega(x, y)=\mathscr{B}(x, y)-\mathscr{B}(y, x), \quad \forall x, y \in A,
$$

is a symplectic form on the associated Jordan algebra $(J(A), \circ)$.

Proof. It follows from (for any $x, y, z \in A$ )

$$
\begin{aligned}
\omega(x \circ y, z)+\text { C.P. }=\mathscr{B}(x \cdot y, z) & -\mathscr{B}(x, y \cdot z) \\
& +\mathscr{B}(y \cdot x, z)-\mathscr{B}(y, x \cdot z)+\text { C.P. }=0,
\end{aligned}
$$

where "C.P." means cyclic permutation.

REMARK 5.16. If a bilinear form $\mathscr{B}$ on a pre-Jordan algebra $(A, \cdot)$ is symmetric, then it is obvious that $\mathscr{B}$ satisfies Eq. (5.10) if and only if $\mathscr{B}$ satisfies Eq. (5.11).

Definition 5.17. Let $(A, \cdot)$ be a pre-Jordan algebra. A skew-symmetric bilinear form $\mathscr{B}$ on $A$ is called invariant if $\mathscr{B}$ satisfies Eq. (5.9). A symmetric bilinear form $\mathscr{B}$ on $A$ satisfying Eq. (5.11) is called a 2-cocycle.

Proposition 5.18. Let $(A, \cdot)$ be a pre-Jordan algebra and $\omega$ be a skewsymmetric bilinear form on A. If $\omega$ is invariant, then $\omega$ is a symplectic form on the associated Jordan algebra $J(A)$. Conversely, if $\omega$ is a nondegenerate symplectic form on a Jordan algebra $(J, \circ)$, then $\omega$ is invariant on the compatible pre-Jordan algebra given by Eq. (4.10).

Proof. If $\omega$ is invariant, then

$\omega(x \circ y, z)=\omega(x \cdot y, z)+\omega(y \cdot x, z)=\omega(y, z \circ x)+\omega(x, y \circ z), \quad \forall x, y, z \in A$.

Thus $\omega$ is a symplectic form on the associated Jordan algebra $J(A)$. The second half part follows from Proposition 4.9 immediately.

From the proof of Proposition 5.18, the following conclusion is obvious.

Corollary 5.19. Let $(A, \cdot)$ be a pre-Jordan algebra. If a skew-symmetric bilinear form $\omega$ is invariant, then $\omega$ satisfies

$$
\omega(x \cdot y, z)+\omega(y \cdot z, x)+\omega(z \cdot x, y)=0, \quad \forall x, y, z \in A .
$$

Corollary 5.20. Let $(A, \cdot)$ be a pre-Jordan algebra and $r \in A \otimes A$. Suppose that $r$ is symmetric and nondegenerate. Then $r$ is a solution of JPequation in $A$ if and only if the bilinearform $\mathscr{B}$ induced by r through Eq. (1.10) is a 2-cocycle on A. 
Proof. It follows from Proposition 5.8 and Proposition 5.14.

Corollary 5.21. Let $(A, \cdot)$ be a pre-Jordan algebra. Then

$$
r=\sum_{i}^{n}\left(e_{i} \otimes e_{i}^{*}+e_{i}^{*} \otimes e_{i}\right)
$$

is a symmetric solution of $J P$-equation in $A \ltimes_{L_{*}^{*}, 0} A^{*}$, where $\left\{e_{1}, \ldots, e_{n}\right\}$ is a basis of $A$ and $\left\{e_{1}^{*}, \ldots, e_{n}^{*}\right\}$ is the dual basis. Moreover, there is a symmetric 2-cocycle $\mathscr{B}$ on $A \ltimes_{L_{,}^{*}, 0} A^{*}$ induced by $r$ through Eq. (1.10) which is given by Eq. (1.11).

Proof. Since id is an $\mathscr{O}$-operator of the pre-Jordan algebra $(A, \cdot)$ associated to the bimodule $(L ., 0, A)$, the conclusion follows from Theorem 5.11 and Corollary 5.20.

There is a "chain" of algebraic equations and bilinear forms on the Jordan algebra and the pre-Jordan algebras corresponding to the algebra relations given in Section 4. It can be interpreted by a simplified illustration as follows.

\begin{tabular}{|c|c|c|}
\hline \multirow{3}{*}{$\begin{array}{l}\text { Symmetric bilinear form } \\
\text { Jordan algebra (invariant) }\end{array}$} & \multirow{3}{*}{ JYBE } & \multirow{2}{*}{$\begin{array}{c}\text { Skew-symmetric bilinear form } \\
\text { Jordan algebra (symplectic) }\end{array}$} \\
\hline & & \\
\hline & & Pre-Jordan algebra (invariant) \\
\hline & & $\downarrow J P$-equation \\
\hline & & Symmetric bilinear form \\
\hline & & Pre-Jordan algebra (2-cocycle) \\
\hline
\end{tabular}

\section{Generalization: Jordan analogues of Loday algebras}

In fact, there are certain algebras structures which are similar to dendriform algebras, such as quadri-algebras of Aguiar and Loday ([4]) and octo-algebras of Leroux ([38]). All of them are called Loday algebras ([49], or ABQR operad algebras in [23], [24]). These algebras have a common property of "splitting associativity", that is, expressing the multiplication of an associative algebra as the sum of a string of binary operations ([41]). Explicitly ([38]), let $(X, *)$ be an associative algebra and $\left(*_{i}\right)_{1 \leq i \leq N}: X \otimes X \rightarrow X$ be a family of binary operations on $X$. Then the operation $*$ splits into the $N$ operations $*_{1}, \ldots, *_{N}$ if

$$
x * y=\sum_{i=1}^{N} x *_{i} y, \quad \forall x, y \in X .
$$


Motivated by the study of Loday algebras, it is natural to generalize the study in this paper in certain wide extent as follows. Let $(X, \circ)$ be a Jordan algebra and $\left(*_{i}\right)_{1 \leq i \leq N}: X \otimes X \rightarrow X$ be a family of binary operations on $X$. Then the operation $\circ$ splits into the commutator of $N$ operations $*_{1}, \ldots, *_{N}$ if

$$
x \circ y=\sum_{i=1}^{N}\left(x *_{i} y+y *_{i} x\right), \quad \forall x, y \in X .
$$

Like Loday algebras, only Eq. (6.2) is too general to get more interesting structures. So some additional conditions for the above operations $*_{i}$ to define what we call Jordan analogues of Loday algebras are necessary. We will pay our main attention to the case that $N=2^{n}, n=0,1,2, \ldots$ As the study on the Loday algebras in such cases given in [9] and generalizing the study in this paper, we define the "rule" of constructing the operations $*_{i}$ to define Jordan analogues of Loday algebras as follows: the $2^{n+1}$ operations give a natural bimodule structure of an algebra with the $2^{n}$ operations on the underlying vector space of the algebra itself, which is the beauty of such algebra structures. That is, by induction, for the algebra $\left(A, *_{i}\right)_{1 \leq i \leq 2^{n}}$, besides the natural (regular) bimodule of $A$ on the underlying vector space of $A$ itself given by the left and right multiplication operators, one can introduce the $2^{n+1}$ operations $\left\{*_{i_{1}}, *_{i_{2}}\right\}_{1 \leq i \leq 2^{n}}$ such that

$$
x *_{i} y=x *_{i_{1}} y+y *_{i_{2}} x, \quad \forall x, y \in A, 1 \leq i \leq 2^{n},
$$

and their left or right multiplication operators can give a bimodule of $\left(A, *_{i}\right)_{1 \leq i \leq 2^{n}}$ by acting on the underlying vector space of $A$ itself.

In particular, when $N=1$, the corresponding algebra $(A, *)$ according to the above rule is exactly a pre-Jordan algebra. Note that for $n \geq 1(N \geq 2)$, in order to make Eq. (6.2) be satisfied, there is an alternative (sum) form of Eq. (6.3)

$$
x *_{i} y=x *_{i_{1}} y+x *_{i_{2}}^{\prime} y, \quad \forall x, y \in A, 1 \leq i \leq 2^{n},
$$

by letting $x *_{i_{2}}^{\prime} y=y *_{i_{2}} x$ for any $x, y \in A$. In particular, in such a situation, it can be regarded as an operation $*$ of a pre-Jordan algebra that splits into the $N=2^{n}(n=1,2, \ldots)$ operations $*_{1}, \ldots, *_{N}$ (pre-Jordan analogues of Loday algebras). That is, a Jordan algebra is given by

$$
x \circ y=x * y+y * x, \quad \forall x, y \in A,
$$

where $(A, *)$ is a pre-Jordan algebra satisfying Eq. (6.1). In this sense, Jordan analogues of Loday algebras are exactly pre-Jordan analogues of Loday algebras with the form (6.1). 
Therefore it is certainly natural to continue and extend the study in the previous Sections to the algebra systems with more operations as Jordan analogues of Loday algebras. In fact, an outline of such a study of Loday algebras by induction was given in [9]. On the other hand, it is also natural to consider the Lie analogues of Loday algebras as the "opposite" of the above Jordan analogues of Loday algebras. In fact, a notion of L-dendriform algebra was introduced in [10] as a Lie algebraic analogue of dendriform algebra.

ACKNOWLEDGEMENTS. We thank the referee for the important suggestions. This work was supported in part by the National Natural Science Foundation of China (10621101, 10921061, 11221091), NKBRPC (2006CB805905) and SRFDP (200800550015, 20120031110022).

\section{REFERENCES}

1. Aguiar, M., Infinitesimal Hopf algebras, pp. 1-29 in: New Trends in Hopf Algebra Theory, Proc. La Falda 1995, Contemp. Math. 267, Amer. Math. Soc., Providence, RI 2000.

2. Aguiar, M., Pre-Poisson algebras, Lett. Math. Phys. 54 (2000), 263-277.

3. Aguiar, M., On the associative analog of Lie bialgebras, J. Algebra 244 (2001), 492-532.

4. Aguiar, M., Loday, J.-L., Quadri-algebras, J. Pure Appl. Algebra 191 (2004), 205-221.

5. Albert, A. A., A structure theory for Jordan algebras, Ann. of Math. (2) 48 (1947), 446-467.

6. Albert, A. A., On a certain algebra of quantum mechanics, Ann. of Math. (2) 35 (1934), $65-73$.

7. Atkinson, F. V., Some aspects of Baxter's functional equation, J. Math. Anal. Appl. 7 (1963), $1-30$.

8. Bai, C., A unified algebraic approach to the classical Yang-Baxter equation, J. Phys. A 40 (2007), 11073-11082.

9. Bai, C., $\mathcal{O}$-operators of Loday algebras and analogues of the classical Yang-Baxter equation, Comm. Algebra 38 (2010), 4277-4321.

10. Bai, C., Liu, L., and Ni, X., Some results on L-dendriform algebras, J. Geom. Phys. 60 (2010), 940-950

11. Baklouti, A., Benayadi, S., Pseudo-euclidean Jordan algebras, arXiv:0811.3702.

12. Baxter, G., An analytic problem whose solution follows from a simple algebraic identity, Pacific J. Math. 10 (1960), 731-742.

13. Burde, D., Left-symmetric algebras, or pre-Lie algebras in geometry and physics, Cent. Eur. J. Math. 4 (2006), 323-357.

14. Cartier, P., On the structure of free Baxter algebras, Adv. in Math. 9 (1972), 253-265.

15. Chapoton, F., Un théorème de Cartier-Milnor-Moore-Quillen pour les bigèbres dendriformes et les algèbres braces, J. Pure Appl. Algebra 168 (2002), 1-18.

16. Chapoton, F., Livernet, M., Pre-Lie algebras and the rooted trees operad, Int. Math. Res. Notices (2001), 395-408.

17. Chari, V., Pressley, A., A Guide to Quantum Groups, Cambridge Univ. Press, Cambridge 1994.

18. Chu, B. Y., Symplectic homogeneous spaces, Trans. Amer. Math. Soc. 197 (1974), 145-159.

19. Chu, C. H., Jordan triples and Riemannian symmetric spaces, Adv. Math. 219 (2008), 20292057.

20. Connes, A., Kreimer, D., Hopf algebras, renormalization and noncommutative geometry, Comm. Math. Phys. 199 (1998), 203-242. 
21. Drinfel'd, V., Hamiltonian structures on Lie groups, Lie bialgebras and the geometric meaning of the classical Yang-Baxter equations, Soviet Math. Dokl. 27 (1983), 68-71.

22. Ebrahimi-Fard, K., Loday-type algebras and the Rota-Baxter relation, Lett. Math. Phys. 61 (2002), 139-147.

23. Ebrahimi-Fard, K., Guo, L., On products and duality of binary, quadratic, regular operads, J. Pure Appl. Algebra 200 (2005), 293-317.

24. Ebrahimi-Fard, K., Guo, L., Coherent unit actions on operads and Hopf algebras, Theory Appl. Categ. 18 (2007), 348-371.

25. Ebrahimi-Fard, K., Manchon, D., and Patras, F., New identities in dendriform algebras, J. Algebra 320 (2008), 708-727.

26. Foissy, L., Les algèbres de Hopf des arbres enracinés décorés II, Bull. Sci. Math. 126 (2002), 249-288.

27. Frabetti, A., Dialgebra homology of associative algebras, C. R. Acad. Sci. Paris (I) 325 (1997), $135-140$.

28. Frabetti, A., Leibniz homology of dialgebras of matrices, J. Pure Appl. Algebra 129 (1998), 123-141.

29. Hou, D., Bai, C., Jordan D-bialgebras and pre-Jordan bialgebras, preprint, 2009.

30. Iordănescu, R., Jordan Structures in Geometry and Physics, Editura Academiei Romane, Bucharest 2003.

31. Jacobson, N., Lie and Jordan triple systems, Amer. J. Math. 71 (1949), 149-170.

32. Jacobson, N., General representation theory of Jordan algebras, Trans. Amer. Math. Soc. 70 (1951), 509-530.

33. Jacobson, N., Structure and Representations of Jordan Algebras, Amer. Math. Soc. Colloq. Publ. 39, Amer. Math. Soc., Providence, RI 1968.

34. Koecher, M., Imbedding of Jordan algebras into Lie algebras I, Amer. J. Math. 89 (1967), 787-816.

35. Koecher, M., Jordan algebras and differential geometry, pp. 279-283 in: Actes du Congrès International des Mathématiciens 1, Proc. Nice 1970, Gauthier-Villars, Paris 1971.

36. Kupershmidt, B. A., What a classical r-matrix really is, J. Nonlinear Math. Phys. 6 (1999), 448-488.

37. Kaup, W., Zaitsev, D., On symmetric Cauchy-Riemann manifolds, Adv. Math. 149 (2000), 145-181.

38. Leroux, P., On some remarkable operads constructed from Rota-Baxter operators, arXiv: math. QA/0311214.

39. Loday, J.-L., Dialgebras, pp. 7-66 in: Dialgebras and Related Operads, Lecture Notes in Math. 1763, Springer, Berlin 2002.

40. Loday, J.-L., Arithmetree, J. Algebra 258 (2002), 275-309.

41. Loday, J.-L., Scindement d'associativité et algèbres de Hopf, pp. 155-172 in: Actes des Journées Mathématiques à la Memoire de Jean Leray, Proc. Nantes 2002, Sémin. Congr. 9, Soc. Math. France, Paris 2004.

42. Loday, J.-L., Ronco, M., Hopf algebra of the planar binary trees, Adv. Math. 139 (1998), 293-309.

43. Loday, J.-L., Ronco, M., Algèbres de Hopf colibres, C. R. Acad. Sci. Paris 337 (2003), 153158.

44. Loos, O., Jordan triple systems, R-spaces, and bounded symmetric domains, Bull. Amer. Math. Soc. 77 (1971), 558-561.

45. Rota, G.-C., Baxter algebras and combinatorial identities I, Bull. Amer. Math. Soc. 75 (1969), 325-329.

46. Schafer, R., An Introduction to Nonassociative Algebras, Dover, New York 1995.

47. Semenov-Tian-Shansky, M. A., What is a classical r-matrix?, Funct. Anal. Appl. 17 (1983), 259-272. 
48. Upmeier, H., Jordan algebras and harmonic analysis on symmetric spaces, Amer. J. Math. 108 (1986), 1-25.

49. Vallette, B., Manin products, Koszul duality, Loday algebras and Deligne conjecture, J. Reine Angew. Math. 620 (2008), 105-164.

50. Zhelyabin, V. N., Jordan bialgebras and their connection with Lie bialgebras, Algebra and Logic 36 (1997), 1-15.

51. Zhelyabin, V. N., Jordan D-bialgebras defined on semisimple Jordan algebras, Preprint 35, Novosibirsk. Gos. Univ, Novosibirsk 1998.

52. Zhelyabin, V. N., Jordan D-bialgebras and symplectic forms on Jordan algebras, Siberian Adv. Math 10:2 (2000), 142-150.

53. Zhelyabin, V. N., On a class of Jordan D-bialgebras, St. Petersburg Math. J. 11 (2000), 589-609.

DEPARTMENT OF MATHEMATICS

YUNNAN NORMAL UNIVERSITY

KUNMING, YUNNAN 650092

P. R. CHINA

and

CHERN INSTITUTE OF MATHEMATICS \& LPMC

NANKAI UNIVERSITY

TIANJIN 300071

P. R. CHINA

E-mail: dongpinghou22@yahoo.com.cn

CHERN INSTITUTE OF MATHEMATICS \& LPMC

NANKAI UNIVERSITY

TIANJIN 300071

P. R. CHINA

E-mail: baicm@nankai.edu.cn
DEPARTMENT OF MATHEMATICS

CALTECH

PASADENA, CALIFORNIA 91125

USA

E-mail: xni@caltech.edu 